The branching ratio of the two modes of decay of $\mathrm{Fm}^{253}$, i.e., E.C./ $\alpha$, was found to be about 8.5 -which gives $\sim 89.5 \%$ decay by electron capture and $\sim 10.5 \%$ by alpha emission. It was not possible to measure the cross section for the $\mathrm{Cf}^{252}(\alpha, 3 n) \mathrm{Fm}^{253}$ reaction because $\mathrm{Fm}^{253}$ could also be produced from other californium isotopes in the target.

A previous publication ${ }^{4}$ on a possible identification of the $\mathrm{Fm}^{253}$ gave the values of $6.85 \pm 0.04 \mathrm{Mev}$ for the alpha-particle energy, and a half-life $>10$ days.

It is a pleasure to thank the crew of the 60 -inch cyclotron for their extremely careful and skillful operation of the machine during the bombardment. We wish to thank Professor Glenn T. Seaborg for his continued interest.

* On leave from the Israel Atomic Energy Commission, Weizmann Institute of Science, Rehovoth, Israel.

1 Thompson, Ghiorso, Harvey, and Choppin, Phys. Rev. 93, 908 (1954).

${ }^{2}$ Harvey, Chetham-Strode, Ghiorso, Choppin, and Thompson, Phys. Rev. 104, 1315 (1956).

${ }^{3}$ Thompson, Harvey, Choppin, and Seaborg, J. Am. Chem. Soc. 76, 6229 (1954); Choppin, Harvey, and Thompson, J. Inorg. and Nuclear Chem. 2, 66 (1956).

${ }^{4}$ Friedman, Gindler, Barnes, Sjoblom, and Fields, Phys. Rev. 102,585 (1956)

\section{Experimental Test of Parity Conservation in Beta Decay*}

\section{S. Wu, Columbia University, New York, New York} AND

E. Ambler, R. W. Hayward, D. D. Hoppes, and R. P. Hudson, National Bureau of Standards, Washington, D. C.

(Received January 15, 1957)

$I^{\mathrm{s}}$ $\mathrm{N}$ a recent paper $^{1}$ on the question of parity in weak interactions, Lee and Yang critically surveyed the experimental information concerning this question and reached the conclusion that there is no existing evidence either to support or to refute parity conservation in weak interactions. They proposed a number of experiments on beta decays and hyperon and meson decays which would provide the necessary evidence for parity conservation or nonconservation. In beta decay, one could measure the angular distribution of the electrons coming from beta decays of polarized nuclei. If an asymmetry in the distribution between $\theta$ and $180^{\circ}-\theta$ (where $\theta$ is the angle between the orientation of the parent nuclei and the momentum of the electrons) is observed, it provides unequivocal proof that parity is not conserved in beta decay. This asymmetry effect has been observed in the case of oriented $\mathrm{Co}^{60}$.

It has been known for some time that $\mathrm{Co}^{60}$ nuclei can be polarized by the Rose-Gorter method in cerium magnesium (cobalt) nitrate, and the degree of polarization detected by measuring the anisotropy of the succeeding gamma rays. ${ }^{2}$ To apply this technique to the present problem, two major difficulties had to be over- come. The beta-particle counter should be placed inside the demagnetization cryostat, and the radioactive nuclei must be located in a thin surface layer and polarized. The schematic diagram of the cryostat is shown in Fig. 1.

To detect beta particles, a thin anthracene crystal $\frac{3}{8}$ in. in diameter $\times \frac{1}{16}$ in. thick is located inside the vacuum chamber about $2 \mathrm{~cm}$ above the $\mathrm{Co}^{60}$ source. The scintillations are transmitted through a glass window and a Lucite light pipe 4 feet long to a photomultiplier (6292) which is located at the top of the cryostat. The Lucite head is machined to a logarithmic spiral shape for maximum light collection. Under this condition, the $\mathrm{Cs}^{137}$ conversion line $(624 \mathrm{kev})$ still retains a resolution of $17 \%$. The stability of the beta counter was carefully checked for any magnetic or temperature effects and none were found. To measure the amount of polarization of $\mathrm{Co}^{60}$, two additional $\mathrm{NaI}$ gamma scintillation counters were installed, one in the equatorial plane and one near the polar position. The observed gamma-ray anisotropy was used as a measure of polarization, and, effectively, temperature. The bulk susceptibility was also monitored but this is of secondary significance due to surface heating effects, and the gamma-ray anisotropy alone provides a reliable measure of nuclear polarization. Specimens were made by taking good single crystals of cerium magnesium nitrate and growing on the upper surface only an additional crystalline layer containing $\mathrm{Co}^{60}$. One might point out here that since the allowed beta decay of $\mathrm{Co}^{60}$ involves a change of spin of

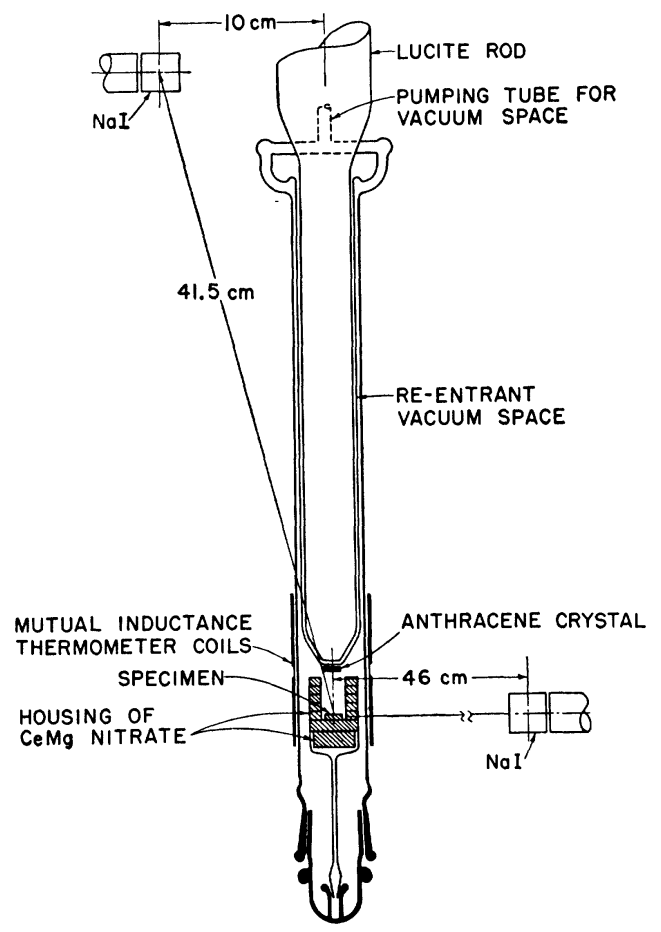

FIG. 1. Schematic drawing of the lower part of the cryostat. 
one unit and no change of parity, it can be given only by the Gamow-Teller interaction. This is almost imperative for this experiment. The thickness of the radioactive layer used was about 0.002 inch and contained a few microcuries of activity. Upon demagnetization, the magnet is opened and a vertical solenoid is raised around the lower part of the cryostat. The whole process takes about $20 \mathrm{sec}$. The beta and gamma counting is then started. The beta pulses are analyzed on a 10-channel pulse-height analyzer with a counting interval of 1 minute, and a recording interval of about 40 seconds. The two gamma counters are biased to accept only the pulses from the photopeaks in order to discriminate against pulses from Compton scattering.

A large beta asymmetry was observed. In Fig. 2 we have plotted the gamma anisotropy and beta asymmetry vs time for polarizing field pointing up and pointing down. The time for disappearance of the beta asymmetry coincides well with that of gamma anisotropy. The warm-up time is generally about 6 minutes, and the warm counting rates are independent of the field direction. The observed beta asymmetry does not change sign with reversal of the direction of the demagnetization field, indicating that it is not caused by remanent magnetization in the sample.

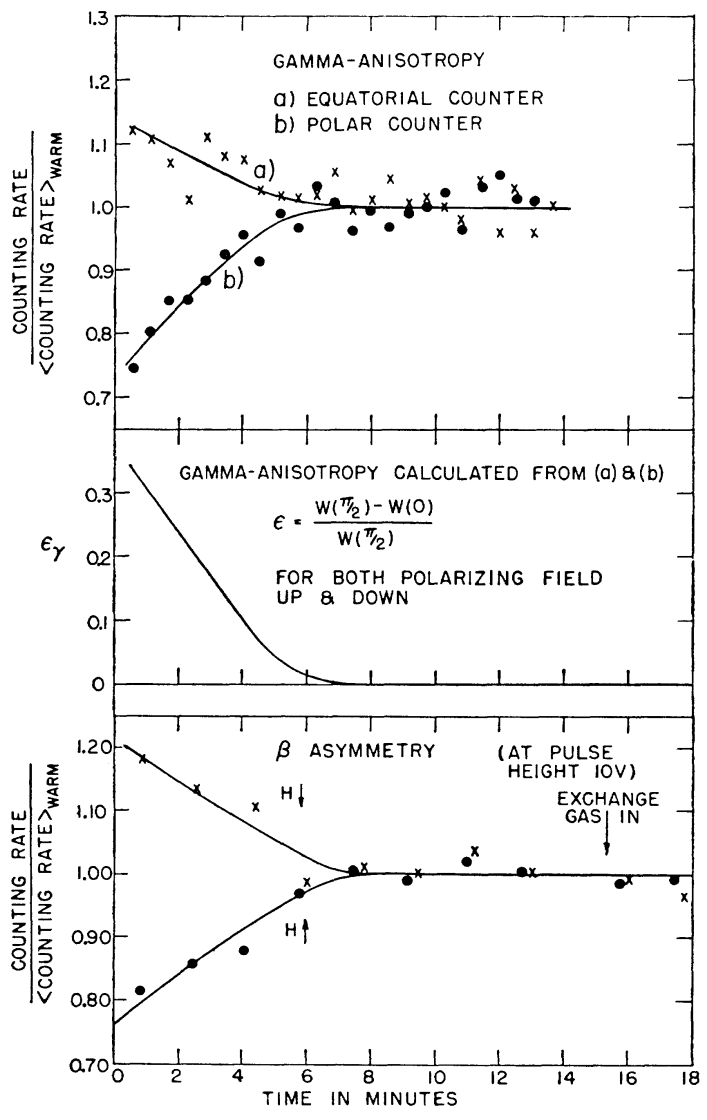

FIG. 2. Gamma anisotropy and beta asymmetry for polarizing field pointing up and pointing down.
The sign of the asymmetry coefficient, $\alpha$, is negative, that is, the emission of beta particles is more favored in the direction opposite to that of the nuclear spin. This naturally implies that the sign for $C_{T}$ and $C_{T}{ }^{\prime}$ (parity conserved and parity not conserved) must be opposite. The exact evaluation of $\alpha$ is difficult because of the many effects involved. The lower limit of $\alpha$ can be estimated roughly, however, from the observed value of asymmetry corrected for backscattering. Al velocity $v / c \approx 0.6$, the value of $\alpha$ is about 0.4 . The value of $\left\langle I_{z}\right\rangle / I$ can be calculated from the observed anisotropy of the gamma radiation to be about 0.6. These two quantities give the lower limit of the asymmetry parameter $\beta\left(\alpha=\beta\left\langle I_{z}\right\rangle / I\right)$ approximately equal to $0 . \bar{\tau}$. In order to evaluate $\alpha$ accurately, many supplementary experiments must be carried out to determine the various correction factors. It is estimated here only to show the large asymmetry effect. According to Lee and Yang $^{3}$ the present experiment indicates not only that conservation of parity is violated but also that invariance under charge conjugation is violated. ${ }^{4}$ Furthermore, the invariance under time reversal can also be decided from the momentum dependence of the asymmetry parameter $\beta$. This effect will be studied later.

The double nitrate cooling salt has a highly anisotropic $g$ value. If the symmetry axis of a crystal is not set parallel to the polarizing field, a small magnetic field will be produced perpendicular to the latter. To check whether the beta asymmetry could be caused by such a magnetic field distortion, we allowed a drop of $\mathrm{CoCl}_{2}$ solution to dry on a thin plastic disk and cemented the disk to the bottom of the same housing. In this way the cobalt nuclei should not be cooled sufficiently to produce an appreciable nuclear polarization, whereas the housing will behave as before. The large beta asymmetry was not observed. Furthermore, to investigate possible internal magnetic effects on the paths of the electrons as they find their way to the surface of the crystal, we prepared another source by rubbing $\mathrm{CoCl}_{2}$ solution on the surface of the cooling salt until a reasonable amount of the crystal was dissolved. We then allowed the solution to dry. No beta asymmetry was observed with this specimen.

More rigorous experimental checks are being initiated, but in view of the important implications of these observations, we report them now in the hope that they may stimulate and encourage further experimental investigations on the parity question in either beta or hyperon and meson decays.

The inspiring discussions held with Professor T. D. Lee and Professor C. N. Yang by one of us (C. S. Wu) are gratefully acknowledged.

* Work partially supported by the U. S. Atomic Energy Commission.

1 T. D. Lee and C. N. Yang, Phys. Rev. 104, 254 (1956)

${ }^{2}$ Ambler, Grace, Halban, Kurti, Durand, and Johnson, Phil. Mag. 44, 216 (1953)

${ }^{3}$ Lee, Oehme, and Yang, Phys. Rev. (to be published). 
${ }^{4}$ Their arguments are as follows: From the $\mathrm{He}^{6}$ recoil experiment and from Eq. (A-4) of reference 1 one concludes that $\left(\left|C_{A}\right|^{2}+\left|C_{A^{\prime}}\right|^{2}\right) /\left(\left|C_{T}\right|^{2}+\left|C_{T^{\prime}}\right|^{2}\right) \leq \frac{1}{3}$. Hence, by comparing Eq. (16) of reference 3 [see also Eq. (A-6) of reference 1], one concludes that the present large asymmetry is possible only if both conservation of parity and invariance under charge conjugation are violated.

\section{Observations of the Failure of Conservation of Parity and Charge Conjugation in Meson Decays : the Magnetic Moment of the Free Muon*}

\author{
Richard L. Garwin, $†$ Leon M. Lederman, \\ and Marcel WeinRich \\ Physics Department, Nevis Cyclotron Laboratories, \\ Columbia University, Irvington-on-Hudson, \\ New York, New York \\ (Received January 15, 1957)
}

$\mathrm{L}$ EE and Yang ${ }^{1-3}$ have proposed that the long held space-time principles of invariance under charge conjugation, time reversal, and space reflection (parity) are violated by the "weak" interactions responsible for decay of nuclei, mesons, and strange particles. Their hypothesis, born out of the $\tau-\theta$ puzzle, ${ }^{4}$ was accompanied by the suggestion that confirmation should be sought (among other places) in the study of the successive reactions

$$
\begin{aligned}
& \pi^{+} \rightarrow \mu^{+}+\nu, \\
& \mu^{+} \rightarrow e^{+}+2 \nu .
\end{aligned}
$$

They have pointed out that parity nonconservation implies a polarization of the spin of the muon emitted from stopped pions in (1) along the direction of motion and that furthermore, the angular distribution of electrons in (2) should serve as an analyzer for the muon polarization. They also point out that the longitudinal polarization of the muons offers a natural way of determining the magnetic moment. ${ }^{5}$ Confirmation of this proposal in the form of preliminary results on $\beta$ decay of oriented nuclei by $\mathrm{Wu}$ et al. reached us before this experiment was begun. ${ }^{6}$

By stopping, in carbon, the $\mu^{+}$beam formed by forward decay in flight of $\pi^{+}$mesons inside the cyclotron, we have performed the meson experiment, which establishes the following facts:

I. A large asymmetry is found for the electrons in (2), establishing that our $\mu^{+}$beam is strongly polarized.

II. The angular distribution of the electrons is given by $1+a \cos \theta$, where $\theta$ is measured from the velocity vector of the incident $\mu$ 's. We find $a=-\frac{1}{3}$ with an estimated error of $10 \%$.

III. In reactions (1) and (2), parity is not conserved.

IV. By a theorem of Lee, Oehne, and Yang, ${ }^{2}$ the observed asymmetry proves that invariance under charge conjugation is violated.

$V$. The $g$ value (ratio of magnetic moment to spin) for the (free) $\mu^{+}$particle is found to be $+2.00 \pm 0.10$.
VI. The measured $g$ value and the angular distribution in (2) lead to the very strong probability that the spin of the $\mu^{+}$is $\frac{1}{2} \cdot{ }^{7}$

VII. The energy dependence of the observed asymmetry is not strong.

VIII. Negative muons stopped in carbon show an asymmetry (also leaked backwards) of $a \sim-1 / 20$, i.e., about $15 \%$ of that for $\mu^{+}$.

IX. The magnetic moment of the $\mu^{-}$, bound in carbon, is found to be negative and agrees within limited accuracy with that of the $\mu^{+} .8$

$\mathrm{X}$. Large asymmetries are found for the $e^{+}$from polarized $\mu^{+}$beams stopped in polyethylene and calcium. Nuclear emulsion (as a target in Fig. 1) yields an asymmetry of about half that observed in carbon.

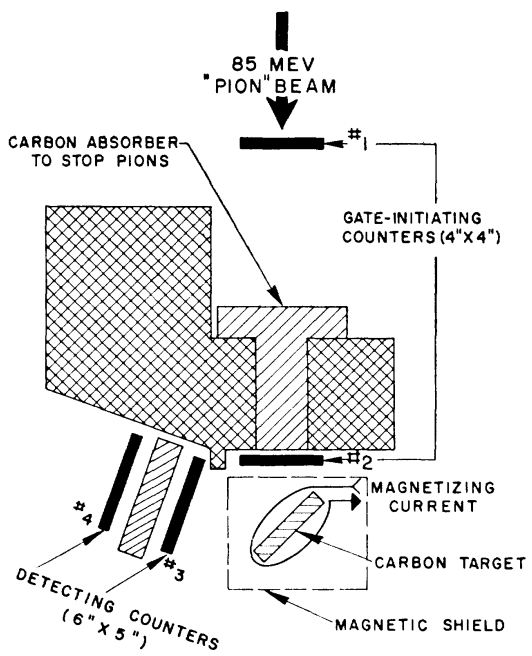

FIG. 1. Experimental arrangement. The magnetizing coil was close wound directly on the carbon to provide a uniform vertical field of 79 gauss per ampere.

The experimental arrangement is shown in Fig. 1. The meson beam is extracted from the Nevis cyclotron in the conventional manner, undergoing about $120^{\circ}$ of magnetic deflection in the cyclotron fringing field and about $-30^{\circ}$ of deflection and mild focusing upon emerging from the 8 -ft shielding wall. The positive beam contains about $10 \%$ of muons which originate principally in the vicinity of the cyclotron target by pion decay-in-flight. Eight inches of carbon are used in the entrance telescope to separate the muons, the mean range of the " $85-$ "Mev pions being $\sim 5$ in. of carbon. This arrangement brings a maximum number of muons to rest in the carbon target. The stopping of a muon is signalled by a fast 1-2 coincidence count. The subsequent beta decay of the muon is detected by the electron telescope 3-4 which normally requires a particle of range $>8 \mathrm{~g} / \mathrm{cm}^{2}(\sim 25-\mathrm{Mev}$ electrons $)$ to register. This arrangement has been used to measure the lifetimes of $\mu^{+}$and $\mu^{--}$mesons in a vast number of elements. ${ }^{9}$ Counting rates are normally $\sim 20$ electrons/ 